\title{
ANÁLISIS DE FENÓMENOS DE LOCALIZACIÓN EN MATERIALES COHESIVO-FRICCIONALES
}

\author{
(ANALYSIS OF STRAIN LOCALIZATION PHENOMENA IN COHESIVE-FRICTIONAL \\ MATERIALS)
}

Francisco J. Martínez Cutillas, Dr. Ingeniero de Caminos ETS Ingenieros de Caminos. Universidad Politécnica de Madrid - España
Director de Tesis: José M. Goicolea Ruigómez

Dr. Ingeniero de Caminos

Fecha de recepción: 24 - VIII - 94

\section{RESUMEN}

El creciente empleo del método de los elementos finitos como berramienta de cálculo estructural requiere la definición de modelos de hormigón capaces de reproducir respuestas más allá del rango eminentemente elástico.

En los últimos años ha crecido fuertemente el interés en la caracterización y modelización de materiales cuyo comportamiento mecánico incluye una fase de reblandecimiento en las etapas próximas a la rotura. En muchos casos dicho reblandecimiento está asociado a la interacción entre los componentes microestructurales del material que genera estados de deformación no homogéneos con una localización de las deformaciones en bandas de pequeño espesor.

El objeto del presente trabajo es contribuir al análisis por métodos numéricos del fenómeno de localización de deformaciones en materiales cohesivo-friccionales. Se dedica especial atención al hormigón en masa y, de manera particular, a la caracterización y modelización de su comportamiento en compresión uniaxial.

\section{Introducción}

El creciente empleo del método de elementos finitos como herramienta de cálculo estructural no ha venido acompañado de un desarrollo de

\section{SUMMARY}

The growth of the finite element method as a tool in structural analysis requires the use of concrete models capable of exhibiting responses beyond the elastic range.

In recent years, considerable interest has arisen in the characterization and modelling of materials which include a softening phase in their mechanical behaviour. This softening is often related to the interaction between microstructural components of the material, which results in the generation of non-homogeneous strain states where deformations are localized in relatively thin bands.

The object of this work is to contribute to the numerical analysis of the phenomenon of strain localization as it arises in cohesivefrictional materials. Special attention is paid to the behaviour of mass concrete and, particularly, to its characterization and modelling during uniaxial compression.

Tesis leída el 15 de julio de 1993 en la Escuela Técnica Superior de Ingenieros de Caminos de Madrid. Obtuvo de calificación APTO CUM LAUDE. 
degradación de las propiedades del hormigón con el nivel de deformación como consecuencia de la fisuración.

Precisamente en los últimos años ha crecido enormemente el interés por la caracterización y modelización de materiales cuyo comportamiento mecánico en las etapas previas a la rotura incluye una fase de reblandecimiento. Las causas que pueden dar lugar a este comportamiento son muy variadas. En metales, la disminución de las propiedades mecánicas con la temperatura, es uno de los motivos de la existencia de respuestas constitutivas con ablandamiento. En materiales granulares densos, el volumen aumenta durante el proceso de deformación, lo que produce una disminución del ángulo de rozamiento y, por tanto, de la capacidad resistente del material; la consecuencia es que las tensiones disminuyen al incrementarse las deformaciones. En arenas flojas saturadas a bajas presiones de confinamiento, el aumento de las presiones intersticiales asociadas al fenómeno de licuefacción ocasiona una disminución de las tensiones efectivas, presentándose un reblandecimiento en la respuesta. En hormigones, la fisuración del mortero y la consiguiente degradación de la estructura interna también es responsable de que disminuya la tensión al aumentar el nivel de deformación.

El reblandecimiento se encuentra a menudo asociado a la existencia de zonas de intensa deformación localizadas en bandas de pequeño espesor, lo que da lugar a estados no homogéneos de deformación. En esta situación es evidente que los ensayos que ayudan a caracterizar el comportamiento mecánico de estos materiales no pueden interpretarse bajo el supuesto de estados uniformes de tensión y deformación en las probetas.

Los modelos disponibles en la actualidad para caracterizar el hormigón no son capaces de predecir correctamente los últimos estadios del comportamiento en compresión uniaxial, en los que se presenta un fuerte reblandecimiento en la respuesta tensión deformación. La pérdida de capacidad resistente en la respuesta se debe fundamentalmente a la degradación de las propiedades mecánicas de los componentes del hormigón dando origen a estados de deformación no uniformes.

Para llegar a caracterizar la respuesta, la investigación llevada a cabo se ha centrado en dos aspectos fundamentales:
- El estudio de elementos capaces de capturar gradientes de deformación elevados, como los que se presentan en las bandas de localización dada la incapacidad de los elementos tradicionales para reproducirlas.

- Una adecuada representación constitutiva de las fases de árido y mortero, de modo que sea posible describir adecuadamente los estadios próximos a la rotura, caracterizados por la iniciación y desarrollo de bandas de localización.

Después de esta presentación se describe brevemente, en primer lugar, un estudio comparativo de diversos elementos formulados mediante métodos variacionales mixtos de deformaciones supuestas y su aptitud para reproducir bandas de localización bajo estados tensionales simples. En segundo lugar, se describe el desarrollo de un modelo de mezclas para representar el comportamiento del hormigón en masa aplicado a diversos ejemplos concretos de compresión uniaxial. Finalmente, se enumeran las conclusiones de este estudio.

\section{Elementos mejorados}

Durante los últimos veinte años se ha dedicado, dentro de la tecnología de elementos finitos, una especial atención al desarrollo de elementos de "altas prestaciones". Dicho término engloba a elementos de bajo orden de interpolación que presentan una gran exactitud en mallas de elementos finitos no excesivamente refinadas. Estos elementos se han propuesto como variantes de los convencionales introduciendo mejoras y ajustes "ad hoc" sin más justificación, muchas veces que la de los propios resultados.

Destacan el cuadrilátero de Wilson y Taylor que corresponde a la modificación del cuadrilátero de interpolación lineal mediante la incorporación de modos de desplazamientos adicionales para mejorar su comportamiento en flexión [17]. Resulta igualmente destacable la formulación volumétrica media propuesta por Nagtegaal et al [6], como método para evitar el bloqueo de elementos cuadriláteros en condiciones próximas a la incompresibilidad. Los conceptos de integración reducida y selectiva así como el planteamiento volumétrico medio son unificados mediante la formulación $\overline{\mathrm{B}}$ por Hughes [3], etc. 
De forma paralela, aunque no simultánea en el tiempo, ha habido un interés en justificar dichos ajustes mediante formulaciones variacionales con el fin de englobarlos dentro de un planteamiento matemáticamente consistente que permitiese ampliar el campo de aplicación para el que inicialmente fueron concebidos. Destacan en este sentido la justificación variacional de la formulación $\overline{\mathrm{B}}$ propuesta por Simó y Hughes [12], así como la formulación más reciente de Simó y Rifai [13] de deformaciones supuestas mejoradas. Esta última formulación engloba de forma natural el planteamiento de modos incompatibles de Wilson y Taylor dentro de un principio variacional de $\mathrm{Hu}-$ Washizu.

Las ventajas fundamentales del planteamiento de elementos mejorados en el contexto de deformaciones supuestas son:

- Permite extender la utilización de elementos que inicialmente fueron concebidos dentro del contexto de la elasticidad lineal al campo de la elasticidad no lineal y la plasticidad.

- La sistematización permite disponer de una estrategia de generación de nuevos elementos.

Estas ventajas han motivado el empleo de los elementos mejorados para evaluar su aptitud para capturar gradientes elevados de deformación y por tanto susceptibles de ser utilizados en problemas de localización de deformaciones.

Se describe, a continuación, primeramente el planteamiento variacional para seguidamente describir la discretización por el método de elementos finitos. Finalmente, se muestran unos ejemplos comparativos de sus prestaciones frente a otros elementos dentro del contexto de localización de deformaciones.

\subsection{Planteamiento del problema variacional}

Se resume en este apartado la formulación de deformaciones supuestas empleada, en el marco desarrollado por Simó y Rifai [13]. Se parte para ello de la formulación variacional de tres campos de $\mathrm{Hu}$-Washizu, para a continuación introducir una reparametrización con base en las deformaciones supuestas. Para un mayor detalle, consultar la referencia citada o la [5].

Sea un cuerpo elástico B que ocupa un dominio $\Omega \subset \mathrm{R}^{3}$. El cuerpo está limitado por una frontera
$S=\partial \Omega$ que se descompone en dos partes, $\mathrm{S}=\mathrm{S}_{\mathrm{d}} \cup \mathrm{S}_{\mathrm{t}}$. Sobre $\mathrm{S}_{\mathrm{d}}$ se conocen los desplazamientos $\mathrm{d}$, mientras que sobre $\mathrm{S}_{\mathrm{t}}$ son conocidas las tensiones $\mathbf{t}$. El vector normal exterior a $\mathrm{S}$ se denota por $\mathbf{n}$. Se considera por tanto:

$$
\begin{aligned}
& \sigma \cdot \mathbf{n}=\mathbf{t} \text { en } S_{t}, \\
& \mathbf{u}=\mathbf{d} \text { en } S_{d}
\end{aligned}
$$

Para cada punto $\mathrm{x} \in \Omega$ se define un campo $\mathbf{b}$ de fuerzas por unidad de volumen. Asimismo se define una función W( $\mathrm{E})$ que corresponde a la densidad de energía interna por unidad de volumen, dependiente del tensor de deformaciones lineal $\varepsilon$. Las incógnitas del problema corresponden al campo de desplazamientos $\mathbf{u}$, al campo de deformaciones infinitesimales $\varepsilon$ y al campo de tensiones $\sigma$ en $\Omega$. La formulación variacional de Hu-Washizu [16] considera los tres campos citados como independientes: $(\mathrm{u}, \boldsymbol{\varepsilon}, \boldsymbol{\sigma}) \in \mathrm{V} \times \boldsymbol{\varepsilon} \times \mathrm{S}$, donde $\mathrm{V}, \boldsymbol{\varepsilon}, \mathrm{S}$ son los espacios funcionales de cuadrado integrable de las funciones solución en desplazamientos, deformación y tensión, respectivamente.

El funcional de Hu-Washizu en función de los tres campos mencionados es:

$$
\mathrm{II}(\varepsilon, \sigma, \mathrm{u})=\mathrm{U}(\varepsilon, \sigma, \mathrm{u})-\mathrm{P}(\mathrm{u})
$$

donde:

$$
\left.\begin{array}{rl}
\mathrm{U}(\varepsilon, \sigma, \mathrm{u}) \stackrel{\text { def }}{=} \int_{\Omega}\left[\mathrm{W}(\varepsilon)+\sigma:\left(\nabla^{\mathrm{s}} \mathrm{u}-\varepsilon\right)\right] \mathrm{dV}, \\
\mathrm{P}(\mathrm{u}) \stackrel{\text { def }}{=} \int_{\Omega} \rho_{0} \mathbf{b} \cdot \mathrm{udV}+\int_{\mathrm{St}} \mathrm{t} \cdot \mathrm{u} \mathrm{dS} .
\end{array}\right\}
$$

Los campos solución $(\mathrm{u}, \boldsymbol{\varepsilon}, \boldsymbol{\sigma})$ serán aquellos para los que el funcional II tome un valor estacionario (mínimo). Igualandu a cero la variación del funcional para valores arbitrarios $(\delta \mathrm{u}, \delta \sigma, \delta \varepsilon)$, se obtienen tres ecuaciones que expresan las condiciones de equilibrio, compatibilidad y constitutivas, respectivamente.

Se admite una reparametrización del campo de deformaciones de la forma:

$$
\varepsilon=\nabla^{s} \mathbf{u}+\bar{\varepsilon} ; \quad \delta \varepsilon=\nabla^{s}(\delta \mathbf{u})+\delta \bar{\varepsilon}
$$

Donde $\nabla^{\mathrm{s}} \mathbf{u}$ (parte simétrica del gradiente de desplazamientos) es la componente "compatible" del campo de deformaciones, y $\bar{\varepsilon}$ es la componente "mejorada" del campo de deformaciones. 


\subsection{Discretización de las ecuaciones variacionales}

\section{Aproximación del campo de deformaciones} compatibles. Para el campo de deformaciones compatibles es posible establecer la aproximación habitual isoparamétrica de elementos finitos. Sea $\mathbf{u}_{\mathrm{e}}^{\mathrm{h}}$ $\in v^{\mathrm{h}} C v_{\text {y }} \nabla^{\mathrm{s}} \mathrm{u}^{\mathrm{h}} \in \nabla^{\mathrm{s}} v^{\mathrm{h}} C \nabla v$, siendo $v^{\mathrm{h}} \mathrm{y}$ $\nabla^{\mathrm{s}} \mathrm{V}^{\mathrm{h}}$ espacios funcionales de dimensión finita, asociados a una discretización "h". La aproximación se realiza mediante las funciones de forma $\mathbf{N}(\xi)$, referidas a coordenadas isoparamétricas $\xi$ :

$$
\begin{aligned}
\mathbf{u}_{\mathrm{e}}^{\mathrm{h}} & =\mathbf{N}_{\mathrm{e}}(\xi) \cdot \mathbf{d}_{\mathrm{e}}, \\
\nabla^{\mathrm{s}} \mathrm{u}_{\mathrm{e}}^{\mathrm{h}} & =\mathbf{B}_{\mathrm{e}}(\xi) \cdot \mathbf{d}_{\mathrm{e}}
\end{aligned}
$$

donde $\mathbf{B}_{\mathrm{e}}(\xi)=\nabla^{\mathrm{s}} \mathbf{N}_{\mathrm{e}}(\boldsymbol{\xi})$ es el operador de interpolación de deformaciones del elemento, y $\mathbf{d}_{\mathrm{e}}$ son los desplazamientos nodales del elemento.

\section{Aproximación del campo de deformaciones} "mejorado". Es posible establecer un criterio de aproximación para $\bar{\varepsilon}$ similar al definido en el campo de deformaciones compatible. Sea $\bar{\varepsilon}_{\mathrm{e}}^{\mathrm{h}}(\xi) \in$ $\bar{\varepsilon}^{\mathrm{h}} \mathrm{C} \bar{\varepsilon}$ :

$$
\bar{\varepsilon}_{\mathrm{e}}^{\mathrm{h}}(\xi)=\mathrm{G}_{\mathrm{e}}(\xi) \cdot \alpha_{\mathrm{e}}
$$

donde $\mathbf{G}_{\mathrm{e}}(\boldsymbol{\xi})$ es el operador de interpolación de deformaciones "mejoradas" del elemento, y $\alpha_{\mathrm{e}}$ son parámetros internos del elemento, generadores del campo de deformaciones "mejoradas".

Si se sustituyen las aproximaciones (6), (7) y (8) en las ecuaciones variacionales se obtiene:

$$
\left.\begin{array}{l}
\sum_{\mathrm{e}=1}^{\text {nel }} \delta \mathbf{d}_{\mathrm{e}}^{\mathrm{T}} \cdot\left[\mathbf{f}_{\mathrm{e}}^{\mathrm{int}}\left(\mathbf{d}_{\mathrm{e}}, \alpha_{\mathrm{e}}\right)-\mathbf{f}_{\mathrm{e}}^{\mathrm{ext}}\right]=0 \\
\sum_{\mathrm{e}=1}^{\text {nel }} \delta \mathbf{d}_{\mathrm{e}}^{\mathrm{T}} \cdot \mathbf{h}_{\mathrm{e}}\left(\mathbf{d}_{\mathrm{e}}, \alpha_{\mathrm{e}}\right)=0
\end{array}\right\}
$$

Estas ecuaciones corresponden en general a un sistema no lineal de ecuaciones cuyas incógnitas son, además de $\mathbf{d}_{\mathrm{e}}$ (las usuales en una formulación en desplazamientos), las variables adicionales $\alpha_{e}$, debidas a la variación independiente de $\bar{\varepsilon}$. La resolución de este sistema se efectúa convencionalmente mediante el procedimiento de Newton-Raphson. Linealizando estas ecuaciones, se obtiene la siguiente ecuación matricial:

$$
\left(\begin{array}{cc}
\mathbf{K}_{\mathrm{e}}^{(\mathrm{k})} & {\left[\Gamma_{\mathrm{e}}^{(\mathrm{k})}\right]^{\mathrm{T}}} \\
\Gamma_{\mathrm{e}}^{(\mathrm{k})} & \mathrm{H}_{\mathrm{e}}^{(\mathrm{k})}
\end{array}\right)\left\{\begin{array}{c}
\Delta \mathbf{d}_{\mathrm{e}} \\
\Delta \alpha_{\mathrm{e}}
\end{array}\right\}=\left\{\begin{array}{c}
-\mathbf{f}_{\mathrm{e}}^{\mathrm{int}(\mathrm{k})}+\mathbf{f}_{\mathrm{e}}^{\mathrm{ext}(\mathrm{k})} \\
-\mathbf{h}_{\mathrm{e}}^{(\mathrm{k})}
\end{array}\right\}
$$

\subsection{Ejemplos comparativos}

Se comenta brevemente en este apartado los resultados obtenidos del análisis comparativo de la respuesta de distintos elementos frente a elementos de formulación convencional ante estados tensionales simples y su comportamiento frente a localización de deformaciones.

Los elementos son cuadriláteros de cuatro nodos que se han formulado dentro del contexto de deformaciones supuestas y que han sido implementados como subrutinas de elemento en el programa de elementos finitos ABAQUS [2]. Estos elementos son: el cuadrilátero de Wilson-Taylor Q1/E4 [13], cuadrilátero de Simó-Rifai Q1/E5 [13], cuadrilátero de Andelfinger-Ramm Q1/E7 [1] y cuadrilátero de Ortiz-Leroy [9].

El primer ejemplo analizado para evaluar la capacidad de estos elementos para capturar gradientes elevados de deformaciones corresponde a un análisis frente a una distribución de tensiones cortantes no uniforme. Para ello se aplica una carga transversal a una de las diagonales y se restringe el

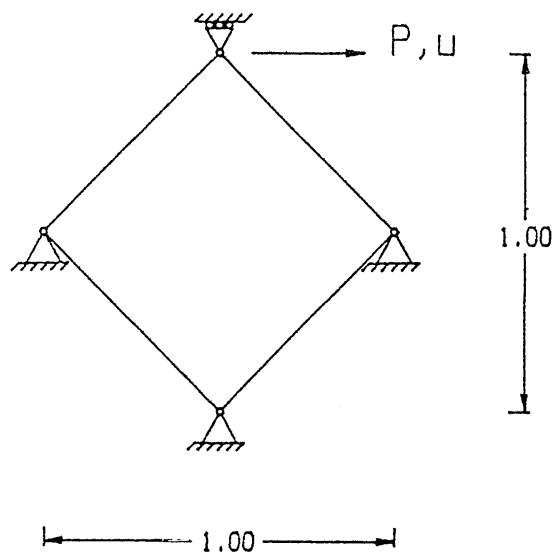

Fig. 1. Problema de cortante no uniforme: Geometria CURVA FUERZA - DESPLAZAMIENTO

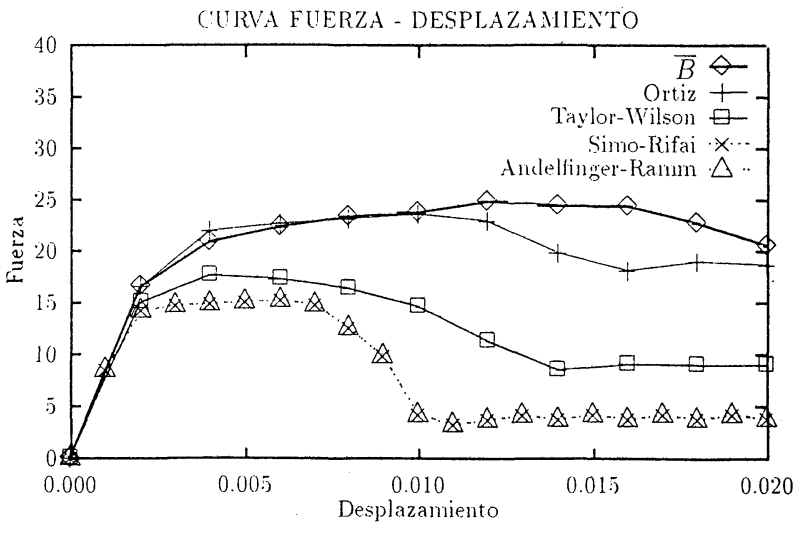

Fig. 2. Problema de cortante no uniforme: Curva fuerza-desplazamiento. 
movimiento de los otros tres nodos como se representa en la Figura 1. Para provocar la localización se ha empleado una ley de ablandamiento parabólica en tensiones y deformaciones plásticas efectivas con un modelo de plasticidad de Von Misses. La Figura 2 muestra la evolución de la fuerza resultante frente al desplazamiento impuesto para los distintos elementos. Se observa una mejor respuesta del elemento de Simó-Rifai y Andelfinger-Ramm frente al elemento convencional $\overline{\mathrm{B}}$, e incluso en comparación con el elemento de Ortiz-Leroy específicamente desarrollado para problemas de localización.

El segundo ejemplo trata de reproducir la formación de una banda de cortante ante la existencia de una distribución periódica de inhomogeneidades. Para representar la falta de homogeneidad se elimina un par de elementos de la diagonal según se representa en la Figura 3. Se ha adoptado un criterio de fluencia tipo Von Misses con módulo de endurecimiento plástico negativo. La distintas deformadas se muestran en la Figura 4. Se aprecia la incapacidad de los elementos $\overline{\mathrm{B}}$ para reproducir

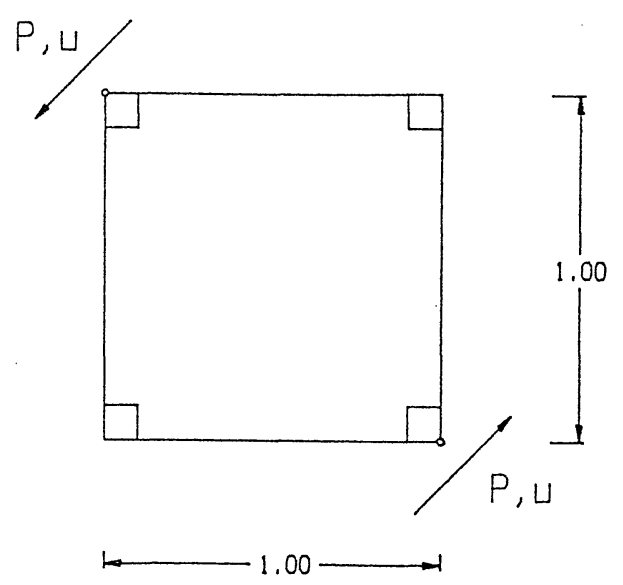

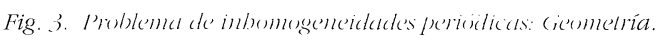

la banda de localización frente a los distintos elementos mejorados. Esta incapacidad se refleja igualmente en el diagrama fuerza-desplazamiento representado en la Figura 5. Dichos élementos no son capaces de reproducir el ablandamiento en la respuesta constitutiva.

Finalmente, el tercer y último ejemplo representa la modelización de una probeta de material granular
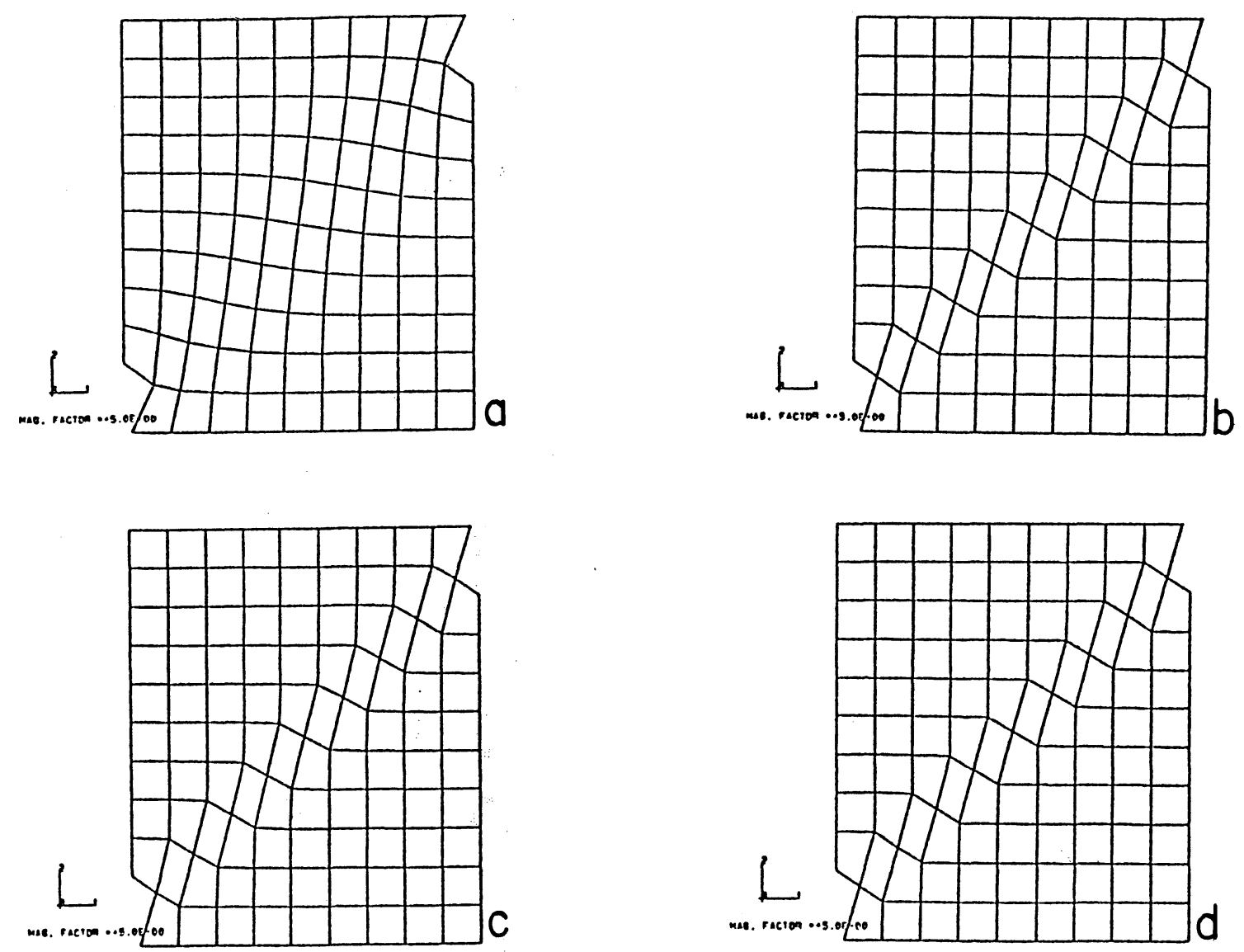

Iing. 4. Problema de inbomogeneidades periódicas. Deformada con elementos: (a) B. (b) Taylor-Wilson. (c) Simó-Rifai. (d) Andelfinger-Ramm. 


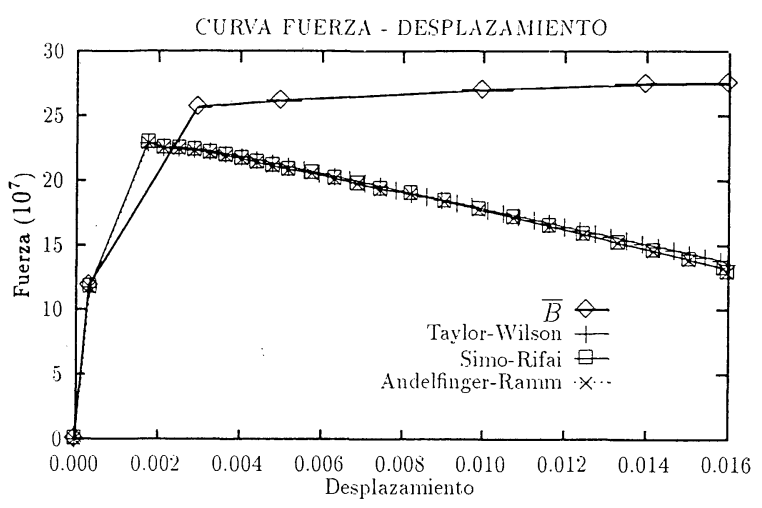

Fig. 5. Problema de inhomogeneidades periódicas: Curva fuerza-desplazamiento.

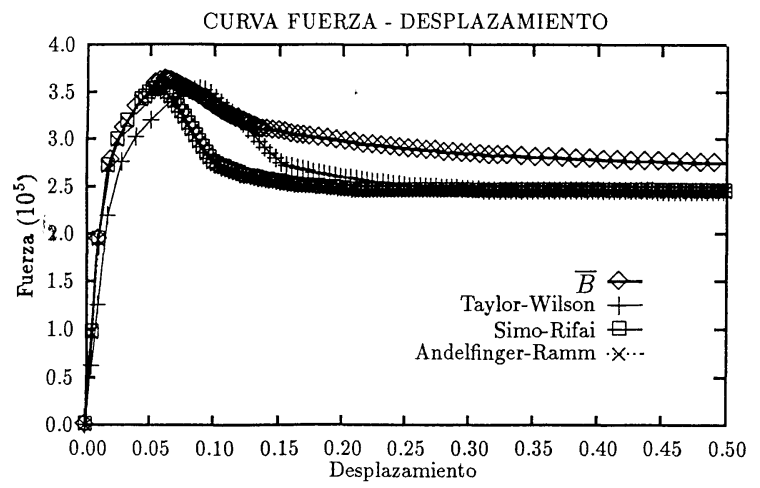

Fig. 7. Problema de compresión con inhomogeneidad: Curva fuerza-desplazamiento.
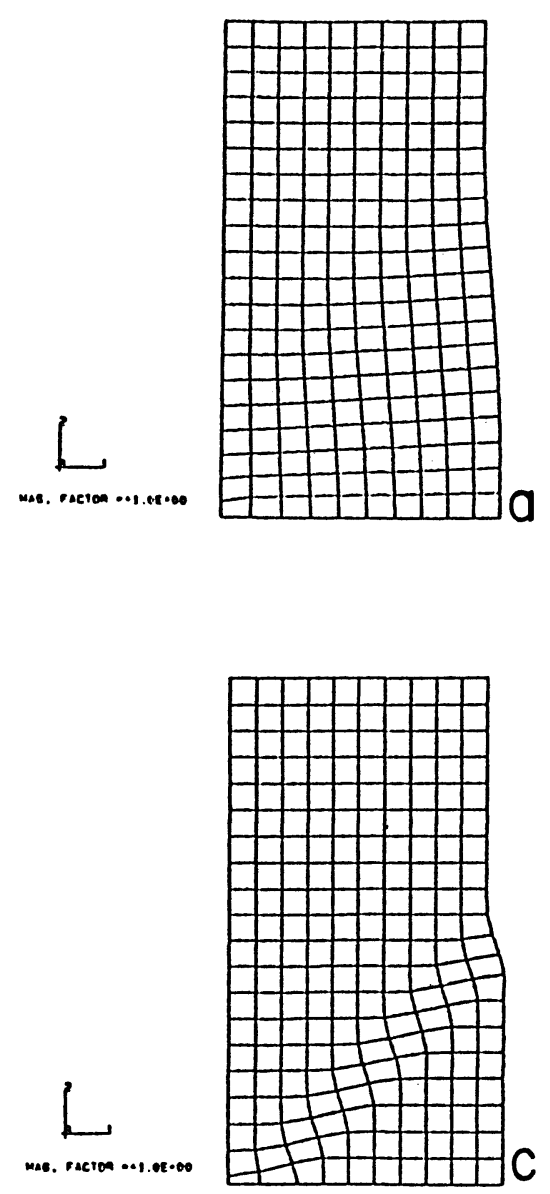
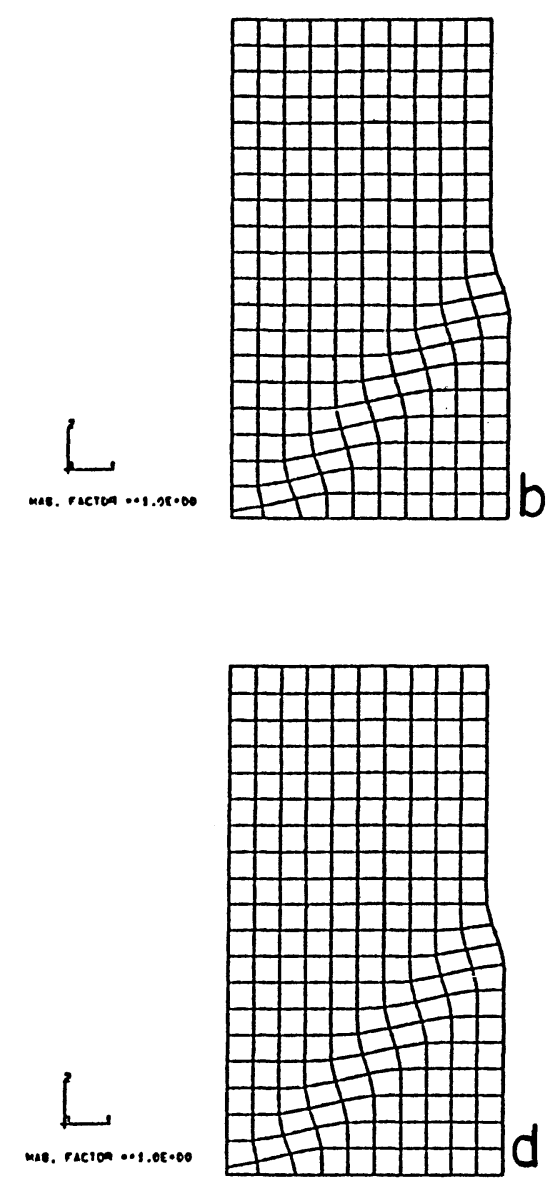

Fig. 6. Problema de compresión con inhomogeneidad. Deformada con elementos: (a) $\bar{B}$. (b) Taylor-Wilson. (c) Simo-Rifai. (d) Andelfinger-Ramm.

con una inhomogeneidad en el centro de la misma. La formulación adoptada corresponde a un modelo elastoplástico no asociativo con función de fluencia Drucker-Prager. La Figura 6 muestra las distintas deformadas obtenidas para distintos elementos. Nuevamente se aprecia que el elemento convencional $\overline{\mathrm{B}}$ no es capaz de reproducir la banda de localización. Análogamente en las curvas fuerzadesplazamiento representadas en la Figura 7 se muestra la respuesta más rígida de los elementos convencionales frente a los mejorados.

\section{Modelización constitutiva del hormigón}

A pesar del enorme potencial que significan los elementos mejorados para reproducir bandas de localización, sólo con la representación correcta de 
la cinemática de la deformación puede resultar insuficiente para modelizar comportamientos constitutivos con fuerte ablandamiento como el que tiene lugar en el hormigón. Por este motivo es preciso ir más allá de la mera modelización del hormigón mediante modelos elastoplásticos convencionales. La razón estriba en que la mayor parte de los aspectos del ablandamiento del hormigón se pueden explicar por su naturaleza heterogénea, al estar compuesto de dos fases: mortero y árido.

La concentración de tensiones que se originan en la interfase árido-mortero generan microdefectos alrededor de los granos. Estos defectos activan un crecimiento de fisuras cuando el hormigón se somete a carga. En las etapas iniciales este proceso de fisuración se encuentra enormemente distribuido, pero para ciertos caminos de carga estas microfisuras se confinan en bandas estrechas que eventualmente pueden dar lugar a planos bien definidos de rotura.

El modelo numérico propuesto en este trabajo pretende verificar que con una adecuada representación del mortero como un material elasto-frágil y los áridos como un material granular y haciendo uso de la teoría de mezclas, es posible representar los modos localizados de deformación en estados tensionales de compresión uniaxial.

\subsection{Hormigón en masa como material compuesto}

La idealización del hormigón en masa como material compuesto fue inicialmente propuesta por Ortiz y Popov [7]. La teoría de mezclas es especialmente adecuada para tener en cuenta la fuerte heterogeneidad del hormigón considerando las dos fases fundamentales: el árido y el mortero. Una consecuencia de esta hipótesis es que para una distribución dada de tensiones aplicadas la redistribución entre cada uno de los constituyentes puede ser muy distinto debido a la diferencia de comportamiento mecánico de los dos componentes. Son precisamente estas tensiones las que deben equilibrarse con las tensiones aplicadas y que difieren mucho entre sí las responsables de los mecanismos inelásticos del comportamiento del hormigón.

La teoría de mezclas se sustenta bajo la hipótesis de que para un volumen arbitrariamente pequeño de hormigón coexisten tanto árido como mortero en fracciones volumétricas fijas $\alpha_{1}$ y $\alpha_{2}$, respectivamente. Las leyes de conservación para una mezcla se pueden obtener requiriendo la invariancia de determinadas propiedades de la ecuación de balance energético (ver Ortiz y Popov [7] y Martínez [5] para más detalle).

Las condiciones de equilibrio se pueden expresar como:

$$
\sigma=\alpha_{1} \sigma_{1}+\alpha_{2} \sigma_{2}
$$

donde $\sigma_{1}$ y $\sigma_{2}$ son respectivamente las tensiones de fase medias del árido y del mortero y $\sigma$ las tensiones globales aplicadas. Conviene hacer notar [8], que $\sigma_{1}$ y $\sigma_{2}$ deben entenderse en el sentido de interacción de continuos; las tensiones de fase son variables macroscópicas correspondientes a una escala de material que es grande en comparación con la microestructura.

La segunda hipótesis corresponde a admitir la ausencia de difusión entre las fases que se establece mediante la compatibilidad de las deformaciones macroestructurales:

$$
\varepsilon \equiv \varepsilon_{1} \equiv \varepsilon_{2}
$$

donde $\varepsilon_{1}, \varepsilon_{2}$ y $\varepsilon$ representan los tensores de deformación macroscópica del árido, mortero y hormigón, respectivamente. Al igual que para las tensiones de fase, para las deformaciones resulta aplicable el mismo comentario, no se pretende que sean medidas de deformación que tengan lugar a nivel microscópico.

Con estas dos hipótesis fundamentales: ley de mezclas (11) y la condición de compatibilidad (12), la relación constitutiva puede ser obtenida a partir de la de sus constituyentes. De acuerdo a [7], [8] y [5] es posible relacionar $\sigma_{1}$ y $\sigma_{2}$ con la tensión $\sigma$ de la siguiente forma:

$$
\begin{aligned}
& \sigma_{1}=B_{1}: \sigma+\rho_{1}, \\
& \sigma_{2}=B_{2}: \sigma+\rho_{2},
\end{aligned}
$$

donde $\mathrm{B}_{1}$ y $\mathrm{B}_{2}$ son los llamados tensores de influencia y $\rho_{1}$ y $\rho_{2}$ las tensiones residuales.

Los modelos propuestos para el árido y el mortero son ambos independientes de la velocidad de deformación. Siguiendo el procedimiento habitual es posible formular una relación incremental tensión-deformación del tipo:

$$
\dot{\sigma}_{1}=\mathrm{D}_{1}^{\mathrm{T}}: \dot{\varepsilon}_{1}, \quad \dot{\sigma}_{2}=\mathrm{D}_{2}^{\mathrm{T}}: \dot{\varepsilon}_{2},
$$


donde $\mathrm{D}_{1}^{\mathrm{T}}$ y $\mathrm{D}_{2}^{\mathrm{T}}$ corresponden a la rigidez tangente del árido y mortero, respectivamente. Haciendo uso de (11) y (12) se obtiene:

$$
\dot{\sigma}=D^{T}: \dot{\varepsilon},
$$

donde:

$$
\mathrm{D}^{\mathrm{T}}=\alpha_{1} \mathrm{D}_{1}^{\mathrm{T}}+\alpha_{2} \mathrm{D}_{2}^{\mathrm{T}}
$$

corresponde a la rigidez tangente del hormigón.

\subsection{Modelización del árido como un material granular}

Los áridos se pueden idealizar como un conjunto de partículas granulares que interactúan por medio de fuerzas de contacto desarrolladas entre sí como en un suelo sin cohesión. El comportamiento constitutivo y la capacidad resistente vienen regidos principalmente por el nivel de presión media. Uno de los criterios de plastificación más extendidos para caracterizar la rotura de suelos sin cohesión es el criterio de rotura de Drucker-Prager que se puede expresar como:

$$
\mathrm{F} \equiv \sqrt{\mathrm{J}_{2}}+\alpha \mathrm{I}_{1}-\mathrm{k}_{\mathrm{p}}
$$

donde $\mathrm{I}_{1}=\sigma_{\mathrm{ii}}$ es el primer invariante del tensor de tensiones $J_{2}=1 / 2 S_{i j} S_{i j}$ es el segundo invariante del tensor desviador de tensiones.

Los coeficientes $\alpha$ y $\mathrm{k}_{\mathrm{p}}$ están relacionados con el ángulo de rozamiento del material $\Phi$ y la cohesión c, respectivamente. Dichas constantes pueden obtenerse imponiendo, por ejemplo, la coincidencia del meridiano de compresión del criterio de Drucker-Prager y Mohr-Coulomb.

Otra característica distintiva de los materiales granulares es el hecho que el ángulo de dilatancia $\Psi$, definido como el cociente entre la componente volumétrica y desviadora de la tasa de deformación plástica, no coincide con el ángulo de rozamiento $\Phi$. Esto implica la definición de una función potencial del tipo:

$$
\mathcal{G} \equiv \sqrt{\mathrm{J}_{2}}+\beta \mathrm{I}_{1}
$$

donde, $\beta$ es una función del ángulo de dilatancia $\Psi$. La tasa de deformación plástica se expresa, por tanto, como:

$$
\varepsilon^{\mathrm{p}}=\dot{\gamma} \frac{\partial \mathcal{G}(\sigma)}{\partial \sigma}
$$

siendo $\dot{\gamma}$ el parámetro de consistencia plástica.

La ley de endurecimiento adoptada para $\alpha$ es una expresión similar a la propuesta por Vermeer y Borst [15] y Leroy y Ortiz [4]:

$$
\left.\begin{array}{c}
\alpha\left(\bar{\varepsilon}_{\mathrm{p}}\right)=\alpha_{0}+\left(\alpha_{\text {sat }}-\alpha_{0}\right) \sqrt{1-\frac{\left[\bar{\varepsilon}_{\mathrm{p}}-\left(\bar{\varepsilon}_{\mathrm{p}}\right)_{\text {sat }}\right]^{2}}{\left(\bar{\varepsilon}_{\mathrm{p}}\right)_{\text {sat }}^{2}}} \bar{\varepsilon}_{\mathrm{p}} \leq\left(\bar{\varepsilon}_{\mathrm{p}}\right)_{\text {sat. }} \\
\alpha\left(\bar{\varepsilon}_{\mathrm{p}}\right)=\alpha_{\text {sat }} \\
\varepsilon_{\mathrm{p}} \geq\left(\bar{\varepsilon}_{\mathrm{p}}\right)_{\text {sat. }}
\end{array}\right\}[21]
$$

Por tanto, el ángulo de rozamiento crece monotónicamente desde $\Phi_{0}$ a $\Phi_{\text {sat }}$ en función de la deformación plástica efectiva.

\subsection{Modelización del mortero como material elasto-frágil}

La fase de mortero es la encargada de proporcionar cohesión a la fase granular. Una característica diferenciadora del hormigón es la degradación de las propiedades elásticas como consecuencia de la microfisuración en el mortero. Esta microfisuración dota al hormigón de un marcado carácter constitutivo anisótropo. En la formulación que se describe a continuación, el mortero se representa como un material elástico en el que se define un criterio para la propagación de la fisuración.

La mayor parte de los modelos de fisura distribuida existentes tienen como inconveniente fundamental la representación correcta de la apertura de fisura en modo I. Se produce una respuesta excesivamente rígida debido a diversos aspectos como: el fenómeno de bloqueo de tensión resultante de la consideración de la continuidad de desplazamientos, la excesiva transferencia de tensiones cortantes a lo largo de la fisura y otros factores de carácter numérico [11].

Una manera de eliminar esta dificultad de la formulación de fisura distribuida es eliminar elementos de la malla. La técnica de eliminación de elementos es un procedimiento para el avance de fisuras que ha sido utilizado con anterioridad por Ortiz y Giannakopoulos [10] en la propagación de fisuras en cerámicas monolíticas en modo mixto. Más recientemente Rots ha empleado una técnica similar para el hormigón [11].

En la formulación que se describe, a continuación se considera que la energía de fractura viene completamente determinada por las condiciones 
existentes en el fondo de la fisura. Por tanto, parece razonable considerar que las condiciones para el crecimiento de una fisura sean función del nivel de tensiones próximas al fondo. Bajo esta premisa postulada por Ortiz y Giannakopoulos [10], un criterio de fractura puede definirse del siguiente modo:

$$
\mathcal{G}^{\mathrm{e}}=\mathcal{G}^{\mathrm{c}}
$$

siendo $\mathcal{G}^{\mathrm{e}}$ la tasa de liberación de energía debido al incremento de una entalla y $\mathcal{G}^{\mathrm{C}}$ es una constante del material denominada tasa crítica de liberación de energía.

Los elementos deben ser eliminados de tal forma que satisfagan el criterio de fractura (22). Considérese, para simplificar, una malla cuadrada de elementos finitos de longitud de elemento $b$ (Figura 8) que contenga una fisura preexistente, sea $\Omega_{\mathrm{e}}$ el dominio de un elemento genérico. La tasa de liberación de energía debido a la eliminación del elemento $\Omega_{\mathrm{e}}$ se puede estimar del siguiente modo:

$$
\mathcal{G}_{\Omega_{\mathrm{e}}}^{\mathrm{e}} \approx \frac{1}{\mathrm{~h}} \int_{\Omega_{\mathrm{e}}} \frac{1}{2} \mathrm{D}_{\mathrm{ijkl}} \varepsilon_{\mathrm{ij}} \varepsilon_{\mathrm{kl}} \mathrm{d} \Omega_{\mathrm{e}} .
$$

El criterio adoptado para eliminar un elemento de la malla es:

$$
\mathcal{G}_{\Omega_{\mathrm{e}}}^{\mathrm{e}} \geq \mathcal{G}^{\mathrm{c}} .
$$

Para elementos cuadrangulares isoparamétricos, la integral en [23] puede aproximarse para un punto de integración en el centroide como:

$$
\mathcal{G}_{\Omega_{\mathrm{e}}}^{\mathrm{e}} \approx \mathrm{W}_{\mathrm{e}} \mathrm{h},
$$

siendo $\mathrm{W}_{\mathrm{e}}=\frac{1}{2} \mathrm{D}_{\mathrm{ijk} k \mathrm{l}} \varepsilon_{\mathrm{ij}} \varepsilon_{\mathrm{kl}}$ la densidad de energía de deformación en el centroide del elemento.

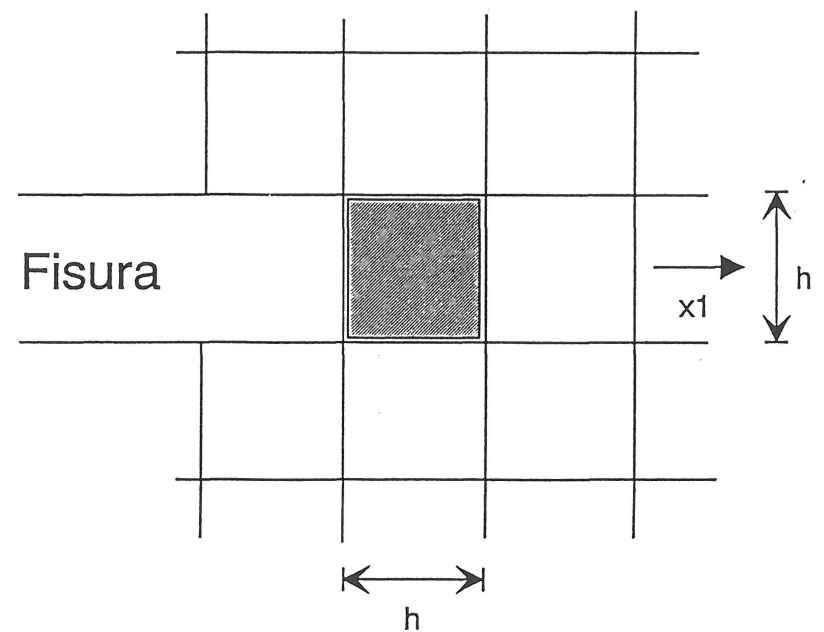

Fig. 8. Modelo de elementos finitos para crecimiento de una fisura.

\section{Resultados numéricos}

El modelo descrito en el apartado anterior ha sido implementado dentro del programa de elementos finitos de propósito general ABAQUS [2] con la intención de efectuar experimentos numéricos. El objetivo final es simular ensayos de diferentes probetas de hormigón con la finalidad de verificar que, con modelos constitutivos simples que representen el mortero y el árido, es posible reproducir el comportamiento en compresión uniaxial y los mecanismos básicos de fallo.

Se ha analizado la respuesta de diversas probetas sometidas a cargas uniaxiales de compresión verificando la influencia del tamaño y de las condiciones de contorno. Los análisis se han efectuado bajo la hipótesis de deformación plana y considerando un tamaño de elemento constante para todo el modelo. De este modo el tamaño del elemento proporciona una longitud característica que representa el tamaño medio del árido. Asimismo se considera la existencia de defectos críticos en la interfaz entre el árido y el mortero que inducen la iniciación del proceso de fisuración.

Desde el punto de vista numérico, ha recibido especial atención, la implementación de la función potencial Drucker-Prager. La dificultad está asociada a la existencia de un vértice en el eje hidrostático, correspondiente a la rotura por cavitación y que conduce a que el problema numérico se encuentre mal condicionado. Se ha adoptado la formulación $\overline{\mathrm{B}}$, de Hughes [3], para prevenir el bloqueo cuando se desarrolla un flujo plástico incompresible. Para el control de fuerzas y desplazamiento se ha considerado el método de longitud de arco modificado de Riks.

Los parámetros que se han considerado para caracterizar el mortero han sido: módulo de Young, $\mathrm{E}_{\text {mortero }}=25 \mathrm{GPa}$, coeficiente de Poisson $\boldsymbol{v}=0,2$; energía de fractura crítica $G^{\mathrm{c}}=160 \mathrm{~J} / \mathrm{m}^{2}$ y fracción volumétrica $\alpha_{\text {mortero }}=0,15$. Los áridos han sido caracterizados por los siguientes parámetros: $\mathrm{E}_{\text {árido }}=40 \mathrm{GPa}, \mathcal{v}=0,2$, ángulo de rozamiento $\Phi_{0}=20^{\circ}, \Phi_{\text {sat }}=30^{\circ}\left(\bar{\varepsilon}_{\mathrm{p}} \geq 0,5 \%\right)$, dilatancia $\Psi=10^{\circ}$, cohesión c=0,4 MPa y fracción volumétrica $\alpha_{\text {árido }}=0,85$.

Se han efectuado diversos análisis modelizando probetas prismáticas de $100 \mathrm{~mm}$ de anchura y de diferentes alturas: 200, 100 y $50 \mathrm{~mm}$, 

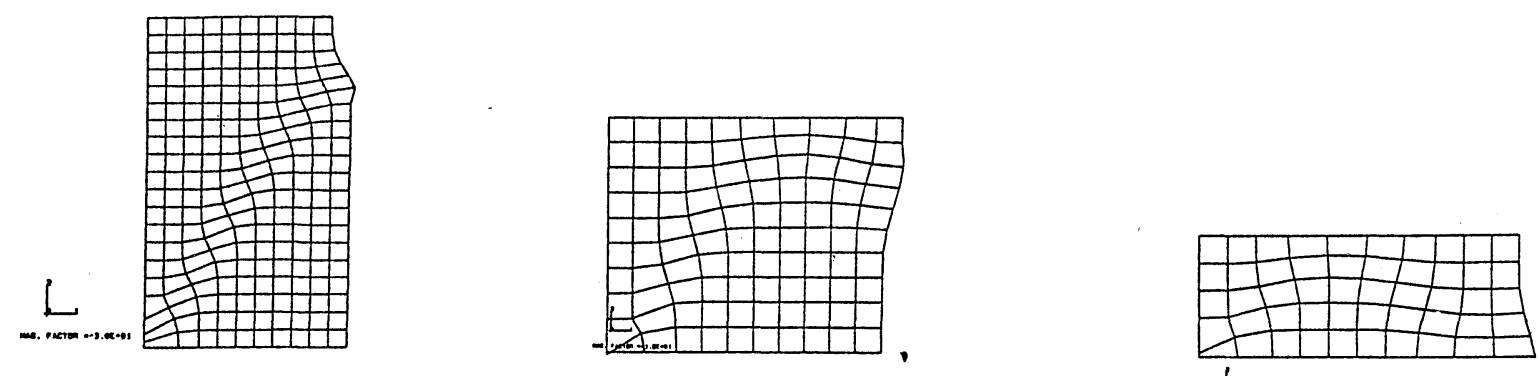

$L$

Fig. 9. Configuraciones deformadas de probetas de distinta esbeltez.

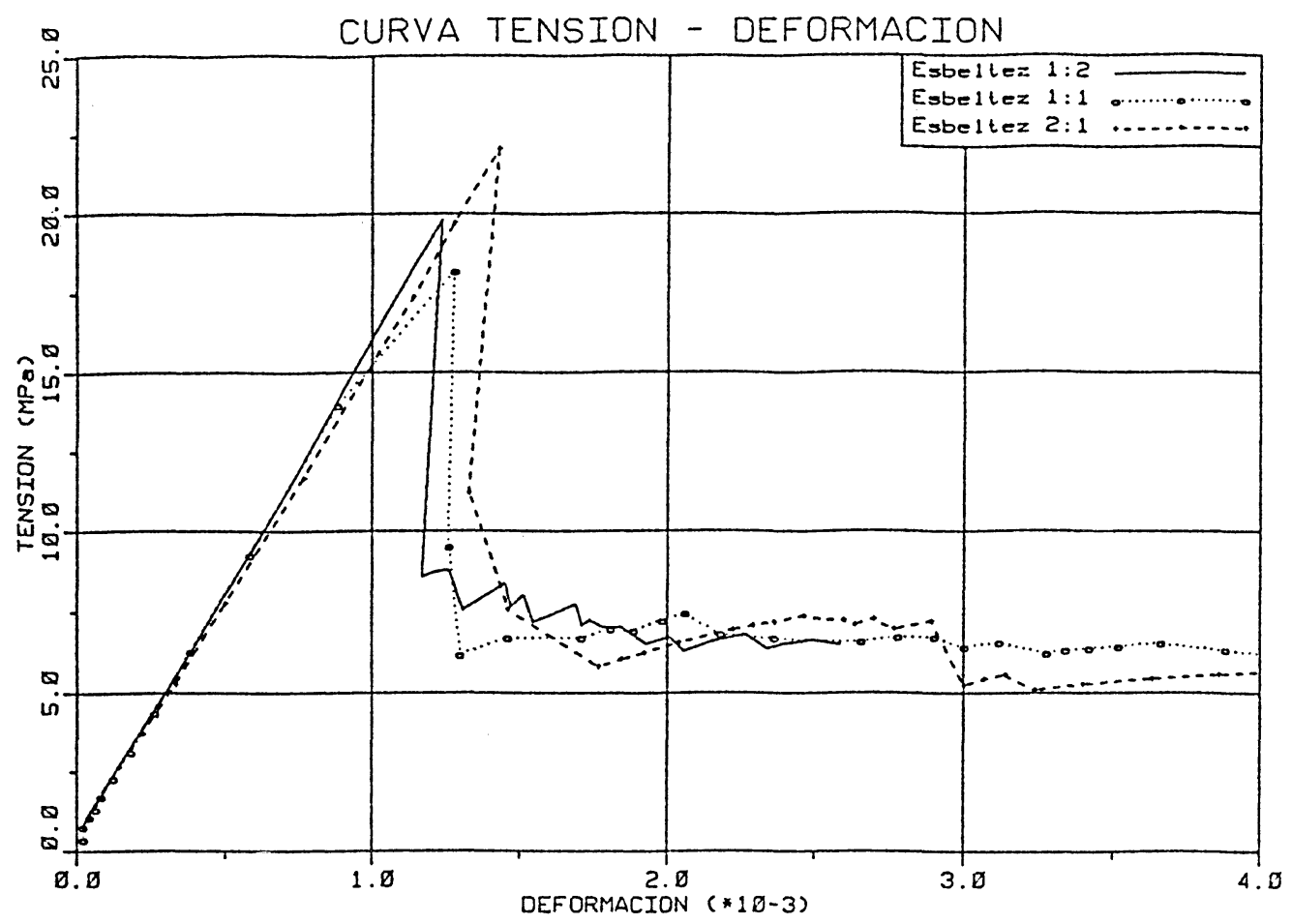

Fig. 10. Curvas tensión-deformación en probetas de distinta esbeltez.

correspondiente a esbelteces de 1:2, 1:1 y 2:1 respectivamente. La Figura 9 representa las configuraciones deformadas de las tres probetas, mostrando bandas de cortante bien definidas en cada caso. El ángulo con la dirección principal de compresión es de $30^{\circ}$ en concordancia tanto cualitativa como cuantitativa con los resultados experimentales obtenidos por Van Mier [14]. Tan pronto como la banda de cortante alcanza la superficie de la probeta, la curva fuerzadesplazamiento sufre una brusca caída experimentando un fuerte ablandamiento en la respuesta como se aprecia en la Figura 10. Es importante hacer notar que como consecuencia de la aparición de la banda de cortante, el campo de tensiones y deformaciones deja de ser uniforme a partir de la tensión de pico.

Diversos análisis se han efectuado con una probeta cúbica de $100 \mathrm{~mm}$ de lado y con diferentes coeficientes de rozamiento entre la probeta y los platos de carga. El objetivo ha sido evaluar numéricamente la influencia de las condiciones de contorno (especialmente el rozamiento) en la respuesta uniaxial en compresión del hormigón. Se han considerado tres coeficientes de rozamiento: 0,2; 0,05; y 0,01. La Figura 11 muestra las configuraciones deformadas para las tres condiciones de contorno y el cambio del ángulo de la banda de cortante cuando alcanza el borde. 

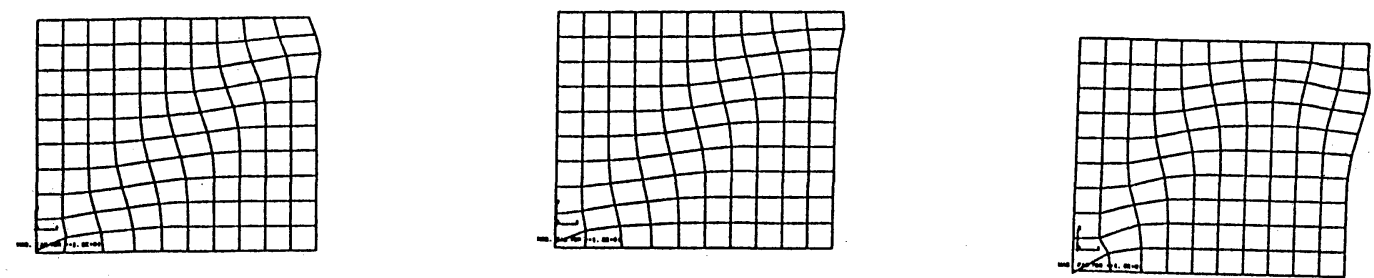

Fig. 11. Configuraciones deformadas en probetas con distintas condiciones de contorno.

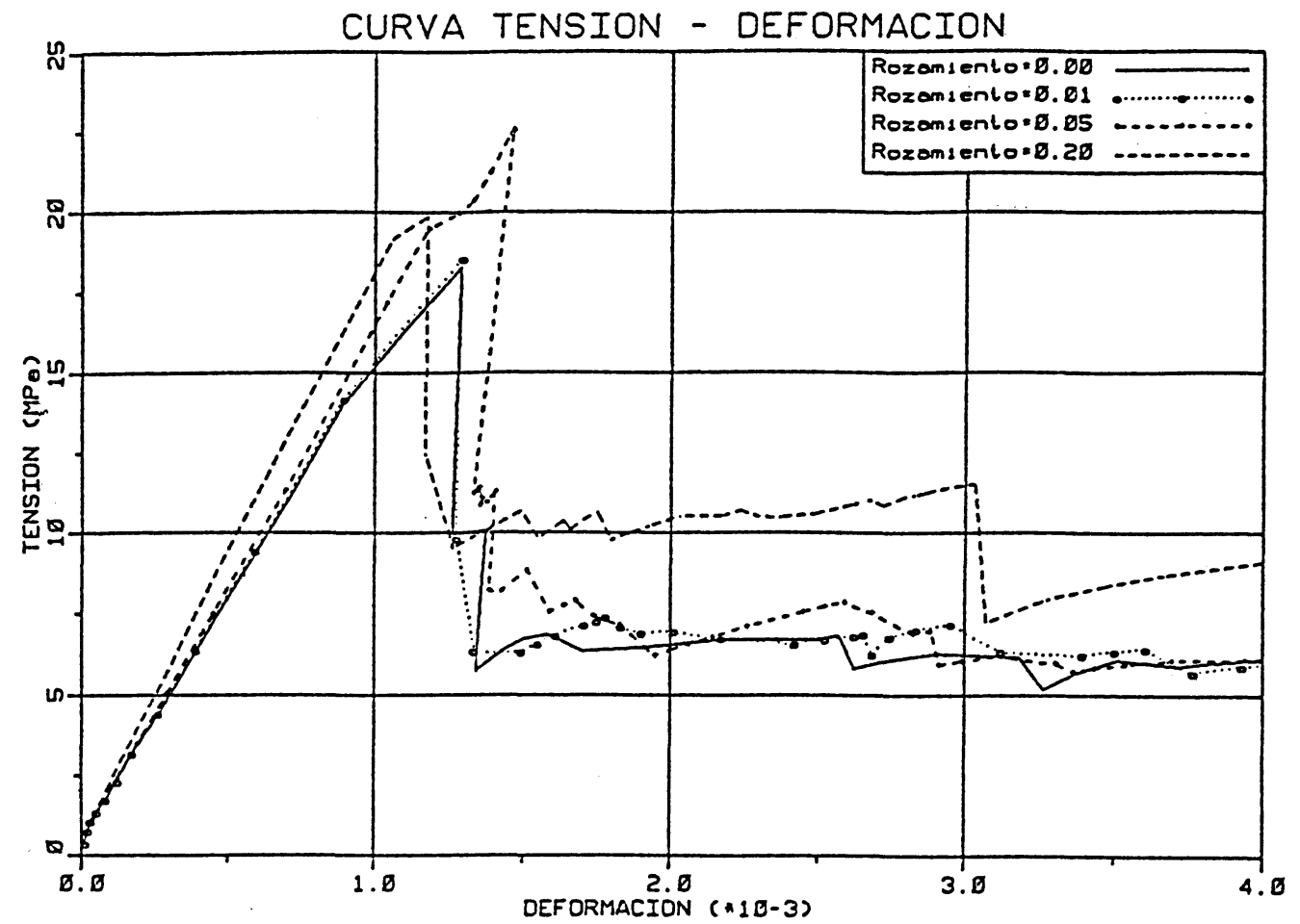

Fig. 12. Curtus tension-deformación én probetas con distintas condiciones de conturno.

Las diferentes curvas fuerza-desplazamiento se recogen en la Figura 12 donde se aprecia un incremento de la tensión de pico con el nivel de rozamiento.

\section{Conclusiones}

Como consecuencia de los resultados obtenidos de la presente investigación, se pueden extraer las siguientes conclusiones:

- El creciente uso del método de los elementos finitos como herramienta de cálculo estructural requiere la disponibilidad de modelos constitutivos, que reproduzcan la respuesta más allá del rango eminentemente lineal del hormigón en masa.

- Es posible un tratamiento numérico adecuado de los problemas de localización de deformaciones siempre que se garantice una adecuada representación de gradientes elevados de deformación. Esto exige que los elementos puedan adoptar la cinemática de las bandas de localización.

- Los elementos tradicionalmente aceptados para análisis no lineales como los cuadriláteros $\overline{\mathrm{B}}$, capaces de tratar problemas de plasticidad incompresible sin bloquearse, se muestran sin 
embargo incapaces de representar adecuadamente la cinemática de las bandas de localización.

- Es posible obtener elementos mixtos de altas prestaciones y de propósito general, mediante los métodos de deformaciones supuestas capaces de una representación adecuada de la localización de deformaciones. Estos elementos engloban tanto los propuestos con anterioridad por Wilson y Taylor para problemas exclusivamente lineales, así como otros desarrollados más recientemente por Simó y Rifai y Andelfinger y Ramm.

- La correcta representación de las bandas de localización puede resultar insuficiente para la representación del comportamiento de materiales, como el hormigón en masa, con un fuerte ablandamiento en su respuesta constitutiva. La razón estriba en que los procesos de localización están íntimamente relacionados con la fisuración de la fase de mortero. Es por ello necesario una correcta caracterización constitutiva.

- El modelo de mezclas propuesto, que representa cada fase del hormigón en masa (árido y mortero) con modelos constitutivos simples, es capaz de representar la fisuración longitudinal y las bandas de cortante en estados de compresión uniaxial.

- El estado de deformación en probetas de hormigón en compresión uniaxial es claramente no homogéneo una vez superada la tensión pico. A pesar de que el modo de fallo es dependiente del tamaño de las probetas y de las condiciones de contorno, las curvas fuerza-desplazamiento no presentan grandes diferencias.

\section{BIBLIOGRAFIA}

[1] Andelfinger, U., y Ramm, E. (1992): EAS-Elements for 2D-3D, plate and shell structures and their equivalence to $H R$ Elements. Submitted to Int. J. Num. Meth. Eng.

[2] Hibbitt, Karlsson \& Sorensen (1989): ABAQUS User's manual. HKS, Inc.

[3] Hughes, T. J. R. (1980): Generalization of selective integration procedures to anisotropic and nonlinear media. Int. J. Num. Meth. Eng, vol. 15, pp. 1413-1418.

[4] Leroy, Y. y Ortiz, M. (1990): Finite element analysis of transient strain localization phenomena in frictional solids. Int. J. for Num. and Analyt. Meth. in Geomech, vol. 14, pp. 93-124.

[5] Martínez, F. (1993): "Análisis de fenómenos de localización de deformaciones en materiales cohesivo-friccionales." Tesis Doctoral. Universidad Politécnica de Madrid.

[6] Nagtegaal, J. C., Parks, D. M. y Rice J. R. (1974): On numerically accurate finite element solutions in the fully plastic range. Comp. Meth. Appl. Mech. Eng. 4, pp. 153-177.

[7] Ortiz, M. y Popov, E. P. (1982): Plain concrete as a composite material. Mech. of Mat. 1, pp. 139-150.

[8] Ortiz, M. (1985): A constitutive theory for the inelastic behavior of concrete, Mech. of Mat, 4, 67.

[9] Ortiz, M., Leroy, Y. y Needleman, A. (1987): A finite element method for localized failure analysis. Comp. Meth. in Appl. Mech. and Eng. 61, pp. 189-214.
[10] Ortiz, M. y Giannakopoulos, A. E. (1990): Crack propagation in monolithic ceramics under mixed mode loading. Int. J. of Fract. 44, pp. 233-258

[11] Rots, J. G. (1992): Removal of finite elements in smeared crack analysis. Proc. 3rd. Int. Conf. on Comp. Plast. Barcelona.

[12] Simó, J. C. y Hughes, T. J. R. (1986): On the variational foundations of assumed strain methods. J. Appl. Mech. ASME, 53, pp. 51-54.

[13] Simó, J. C. y Rifai, M. S. (1990): A class of mixed assumed methods and the method of incompatible modes. Int. J. Num. Meth. Eng, vol. 29, pp. 1595-1638.

[14] Van Mier, J. G. (1984): Strain softening of concrete under multiaxial loading conditions. Phd Thesis. Technische Hogeschool Eindhoven.

[15] Vermeere, P. A. y Borst, R. (1984): Non-associated plasticity for soils, concrete and rocks. Heron, vol. 29, núm. 3, Delft.

[16] Washizu, K. (1968): Variational Metbods in Elasticity and Plasticity. Pergamon Press. Oxford.

[17] Wilson, E. L., Taylor, R. L., Doherty, W. P. y Ghaboussi, J. (1973): Incompatible displacement models. Núm. and Comp. Meth. in Struct. Mech. (eds. S. T. Fenves et al), pp. 43-57. Academic Press. 